\title{
Trends in the prevalence of asthma and allergic rhinitis in Italy between 1991 and 2010
}

\author{
R. de Marco*, V. Cappa*, S. Accordini*, M. Rava*, L. Antonicelli", O. Bortolami*, \\ M. Braggion*, M. Bugiani ${ }^{\ddagger}$, L. Casali ${ }^{+}$, L. Cazzoletti*, I. Cerveri ${ }^{\S}$, A.G. Fois ${ }^{f}$, \\ P. Girardi*, F. Locatelli*, A. Marcon*, A. Marinoni**, M.G. Panico ${ }^{\# \#, ~ P . ~ P i r i n a ~}{ }^{f}$, \\ S. Villani**, M.E. Zanolin* and G. Verlato* for the GEIRD study group
}

ABSTRACT: The prevalence of asthma increased worldwide until the 1990s, but since then there has been no clear temporal pattern.

The present study aimed to assess time trends in the prevalence of current asthma, asthma-like symptoms and allergic rhinitis in Italian adults from 1990 to 2010.

The same screening questionnaire was administered by mail or phone to random samples of the general population (age 20-44 yrs) in Italy, in the frame of three multicentre studies: the European Community Respiratory Health Survey (ECRHS) (1991-1993; $n=6,031$ ); the Italian Study on Asthma in Young Adults (ISAYA) (1998-2000; $n=18,873)$; and the Gene Environment Interactions in Respiratory Diseases (GEIRD) study (2007-2010; $n=10,494)$. Time trends in prevalence were estimated using Poisson regression models in the centres that repeated the survey at different points in time.

From 1991 to 2010, the median prevalence of current asthma, wheezing and allergic rhinitis increased from $4.1 \%$ to $6.6 \%$, from $10.1 \%$ to $13.9 \%$ and from $16.8 \%$ to $25.8 \%$, respectively. The prevalence of current asthma was stable during the 1990s and increased (relative risk 1.38, 95\% CI 1.19-1.59) from 1998-2000 to 2007-2010, mainly in subjects who did not report allergic rhinitis. The prevalence of allergic rhinitis has increased continuously since 1991.

The asthma epidemic is not over in Italy. During the past $20 \mathrm{yrs}$, asthma prevalence has increased by $38 \%$, in parallel with a similar increase in asthma-like symptoms and allergic rhinitis.

KEYWORDS: Allergic rhinitis, asthma, epidemiology, prevalence, temporal trends, wheezing

A sthma is the most common chronic respiratory disease in the world [1] and is a significant cause of morbidity worldwide. Approximately 300 million people in the world currently have asthma [2]. The prevalence of asthma increased worldwide in the second half of the last century until the 1990s, but since then, there has been no clear temporal pattern [3].

In children, the prevalence of asthma is still increasing in the countries where the prevalence had been low and has decreased or levelled off in several westernised countries where the prevalence had been high [4]. However, in some industrialised countries, opposite trends have also been found $[5,6]$.

The evidence that there has been a change in asthma prevalence in adults is conflicting, with some studies suggesting that asthma prevalence has stabilised or even decreased [7-10], while others suggest that it is still increasing $[11,12]$.

While the recent trend in asthma prevalence is uncertain, all studies agree that the prevalence of allergic rhinitis, a condition strongly associated with asthma [13], is still on the increase [4, 7, 8, 14-16].

A reliable assessment of the trend of asthma prevalence requires repeated cross-sectional studies on different occasions on the same population, using the same methods over an adequate period of time. Unfortunately, very few studies with these characteristics [17] are available.

Repeated cross-sectional surveys with identical study design and ascertainment methods were
AFFILIATIONS

*Unit of Epidemiology and Medical Statistics, University of Verona, Verona,

\#Allergy Unit, Dept of Internal Medicine, Immuno-Allergic and Respiratory Diseases, Ospedali Riuniti di Ancona, Ancona, -Unit of Respiratory Medicine, National Health Service, Turin, ${ }^{+}$Dept of Internal Medicine, Section of Respiratory Disease, University of Perugia, Perugia,

${ }^{\S}$ Division of Respiratory Diseases, Istituto di Ricovero e Cura a Carattere Scientifico "San Matteo" Hospital Foundation,

${ }^{* *}$ Dept of Health Sciences, Section of Epidemiology and Medical Statistics, University of Pavia, Pavia, fInstitute of Respiratory Diseases, University of Sassari, Sassari,

\#\#Epidemiology Unit, National Health Service, Salerno, Italy. "For a list of the members of the GEIRD study group, see the Acknowledgements.

\section{CORRESPONDENCE}

R. de Marco, Unit of Epidemiology and Medical Statistics, Dept of Public Health and Community Health, University of Verona, c/0 Istituti Biologici II, Strada Le Grazie 8, 37134 Verona, Italy E-mail: roberto.demarco@univr.it

Received: April 082011

Accepted after revision: July 222011 First published online: Oct 172011 
carried out among random samples of young adults (2044 yrs) in several Italian areas between 1991 and 2010.

The present study is aimed at quantifying the temporal change in the prevalence of asthma and allergic rhinitis in the past $20 \mathrm{yrs}$ in the adult population of Italy, a country that is characterised by a medium-to-low level of asthma prevalence [18].

\section{METHODS}

\section{Study samples and design}

Between 1991 and 2010, three repeated multicentre crosssectional surveys on respiratory diseases were carried out by the same research team on random samples of young adults, using the same design.

The first study was the Italian arm of the European Community Respiratory Health Survey (ECRHS), which was carried out between 1991 and 1993 [19]. A sample of the general population aged 20-44 yrs (male:female ratio 1:1) was randomly selected from the registry of the local health authority in each of three Italian centres (3,000 in Turin and Verona, 1,000 in Pavia). All the eligible subjects were sent a postal screening questionnaire (up to three times in the case of non-response). A final phone interview was carried out to reach the remaining non-responders.

7 yrs later (1998-2000) the Italian Study on Asthma in Young Adults (ISAYA) [20] was carried out following the same design, targeting the same population, using the same sampling frame and the same protocol of questionnaire administration as the ECRHS. About 3,000 subjects aged 2044 yrs were randomly selected from the general population in each of nine Italian centres: Ferrara, Pavia, Pisa, Sassari, Sassuolo, Siracusa, Turin, Udine and Verona.

The third study, the Gene Environment Interactions in Respiratory Diseases (GEIRD) study [21], was conducted from 2007 to 2010, applying the same procedures as ECRHS and ISAYA. A random sample of about 3,000 subjects from the general population aged 20-44 yrs was randomly selected in each of seven centres: Ancona, Pavia, Terni, Salerno, Sassari, Turin and Verona.

Overall, 12 Italian centres were involved in at least one study and four in at least two studies. Six centres are located in northern Italy and belong to the Sub-Continental climatic area (Udine, Verona, Pavia, Turin, Ferrara and Sassuolo) and the other six are located in central or southern Italy and belong to the Mediterranean climatic area (Pisa, Ancona, Terni, Sassari, Salerno and Siracusa) (fig. 1). The Sub-Continental region is characterised by a lower average annual temperature and a higher annual temperature range than the Mediterranean one.

Ethical approval was obtained in each centre from the appropriate ethics committee. All participants were fully informed about all aspects of the research project and consented to complete and return the questionnaire

\section{Screening questionnaire, respiratory outcomes and potential confounders}

The questionnaire used in the ISAYA [22] and GEIRD studies (available at www.geird.org) was the same as the one used in the ECRHS [18], with additional questions on the history of asthma, chronic bronchitis, eczema, dyspnoea, smoking habits, self-reported annoyance due to air pollution, and direct and indirect costs of asthma or other breathing problems.

Based on the answers to the questionnaire, a subject was considered to have: 1) current asthma if she/he answered affirmatively at least one of the following questions: "Have you had an attack of asthma in the last 12 months?" and "Are you currently (in the last 12 months) taking any medicine (including inhalers, aerosol and/or tablets) for asthma?"; 2) allergic rhinitis if she/he gave a positive answer to the question: "Do you have any nasal allergies, including hay fever?"; and 3) asthma-like symptoms if she/he gave a positive answer to the questions: "Have you had wheezing or whistling in your chest at any time in the last 12 months?", "Have you woken up with a feeling of tightness in your chest at any time in the last 12 months?" and "Have you been woken up by an attack of shortness of breath at any time in the last 12 months?".

Sex, age, season at the time of the interview (spring, summer, autumn and winter) and type of contact (postal waves and telephone interview) were considered as potential confounders. Moreover, as the centres had different final response rates, each subject was attributed the centre-specific cumulative response percentile rank in which she/he had answered. For this purpose, in each centre and for each study, subjects were arranged according to the date the questionnaire had been filled in and their percentile rank was expressed as a percentage of the total number of eligible subjects. In short, the percentile rank is a function of the promptness of response, which is one of the main potential confounders associated with non-response $[19,23]$. In fact, if the tendency to respond is associated with respiratory health, the crude estimate of prevalence is biased (i.e. early responders may report more symptoms than late or non-responders). Similar methods were used in previous papers $[20,24]$.

\section{Statistical analysis}

Categorical data were summarised as counts with percentages and continuous data as means $\pm \mathrm{SD}$. Comparisons of variables across studies were performed by the Pearson Chi-squared test or by the Kruskal-Wallis test as appropriate.

Adjusted prevalence rates of respiratory outcomes, stratified by study, climatic area and centre, were obtained through a logistic regression model with respiratory outcomes as dependent variables (present versus absent) and sex, age, percentile rank of cumulative response, type of contact and season at the interview as covariates.

To study the change over time of the prevalence of respiratory outcomes, only the four centres participating in at least two out of the three studies were considered (Pavia, Sassari, Turin and Verona). Two Poisson regression models [25] (with robust standard errors) were fitted to the data, using the respiratory outcomes as the dependent variables, and centre plus the other potential confounders as independent variables. The first model addressed the changes in prevalence occurring between ECRHS (1991-1993) and ISAYA (1998-2000), while the second model focused on the changes in prevalence occurring between ISAYA (1998-2000) and GEIRD (2007-2010). Data from three centres (Pavia, Turin and Verona) were available for the first model, while the second model could take into account 


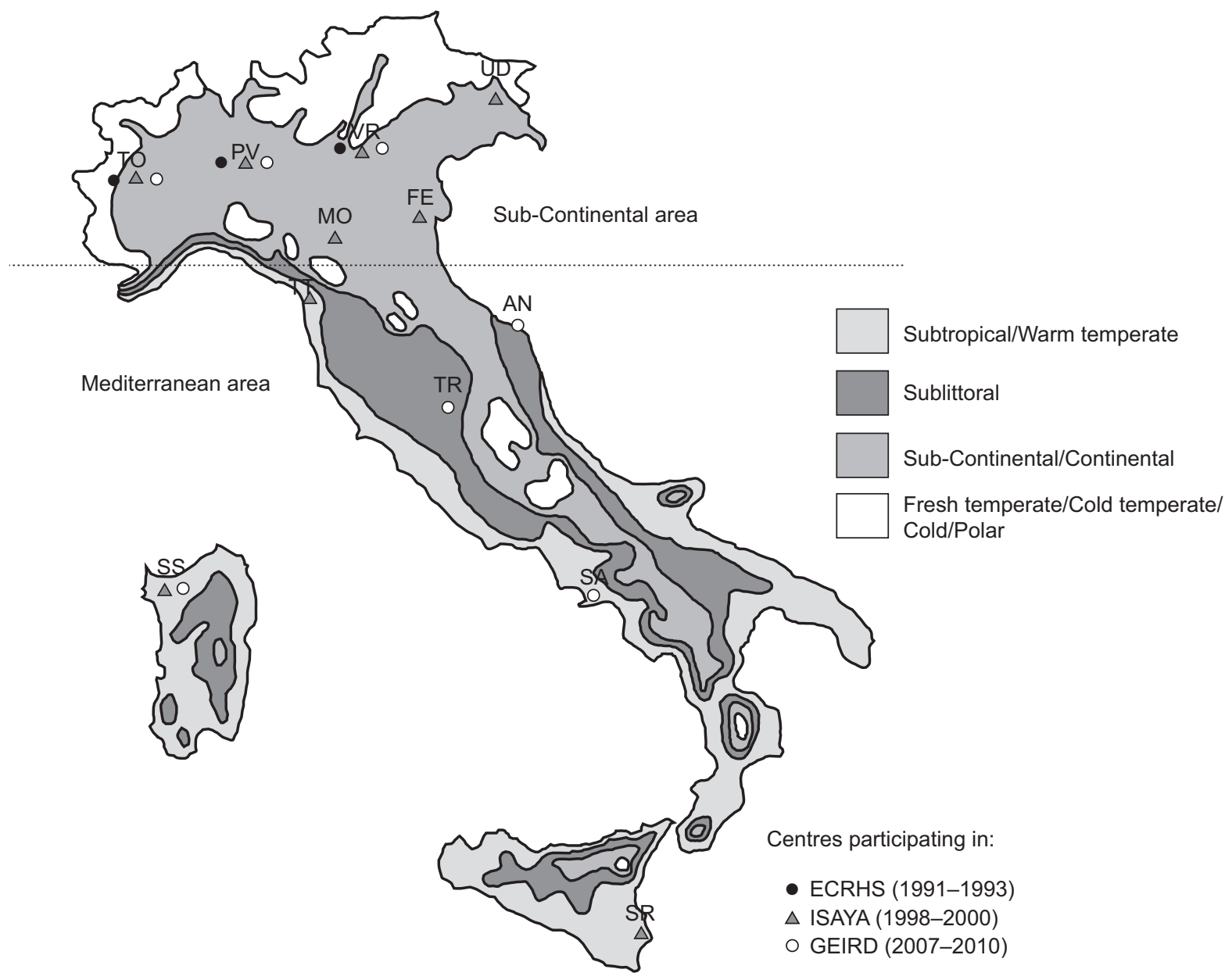

FIGURE 1. Italian centres that participated in the European Community Respiratory Health Survey (ECRHS) (1991-1993), the Italian Study on Asthma in Young Adults (ISAYA) (1998-2000) and the Gene Environment Interactions in Respiratory Diseases (GEIRD) study (2007-2010) surveys, according to climatic regions. UD: Udine; VR: Verona; PV: Pavia; TO: Turin; FE: Ferrara; MO: Sassuolo; PI: Pisa; AN: Ancona; TR: Terni; SA: Salerno; SS: Sassari; SR: Siracusa.

an additional centre (Sassari). The results were expressed as the relative risk (RR) and 95\% confidence interval $(95 \% \mathrm{CI})$ of reporting respiratory outcomes over the two subsequent calendar periods: 1998-2000 versus 1991-1993 and 2007-2010 versus 1998-2000.

\section{Sensitivity analysis}

Differences in the response rates could bias estimates of the change in prevalence over time. To assess the sensitivity of the results to this potential source of bias, the main analyses (Poisson regression models), were repeated after we had truncated the observations at different cumulative response rates $(15 \%, 30 \%, 45 \%, 60 \%, 75 \%$ and $90 \%)$ in each centre and each study. As an example, in the first set of sensitivity analyses, subjects with a cumulative response percentile rank greater than $15 \%$ were excluded. In general, this analysis makes it possible to estimate the time trends when the response rate is the same in different periods of time.

\section{RESULTS}

\section{Response rates and subject characteristics}

Between 1991-1993 and 2007-2010, 35,398 subjects in 12 Italian centres answered the screening questionnaire. The average response rate showed a statistically significant $(\mathrm{p}<0.001)$ decrease (table 1) from $86.2 \%$ in 1991-1993 (ECRHS) to $72.7 \%$ in 1998-2000 (ISAYA) and to 57.2\% in 2007-2010 (GEIRD). The percentage of females (table 2 ) and the mean age of responders increased slightly during the study period $(\mathrm{p}<0.001)$. The percentage of responders who answered the phone interview decreased from $32.9 \%$ to $11.8 \%$ in the same period $(\mathrm{p}<0.001)$. This decrease was mainly due to the increase in the number of mobile phone users and the decrease in landline users. The percentage of current smokers in the total sample decreased significantly from $33.5 \%$ to $27.7 \%$ in the last 10 yrs $(p<0.001)$.

\section{Prevalence of current asthma, allergic rhinitis and asthma- like symptoms in Italy}

The median of the adjusted prevalence rates of current asthma, allergic rhinitis and asthma-like symptoms (table 3) was in general higher in centres in the Mediterranean area than in those in the Sub-Continental area, and increased from 19911993 to 2007-2010 in both regions. Overall, in the last decade, the national median prevalences of asthma and allergic rhinitis increased from $4.6 \%$ to $6.6 \%$ and from $19.4 \%$ to $25.8 \%$, respectively. A similar consistent trend was also observed for asthma-like symptoms. 
TABLE 1 Number of participating subjects and response rates to the screening questionnaire by centre, in the three surveys carried out in Italy between 1991 and 2010

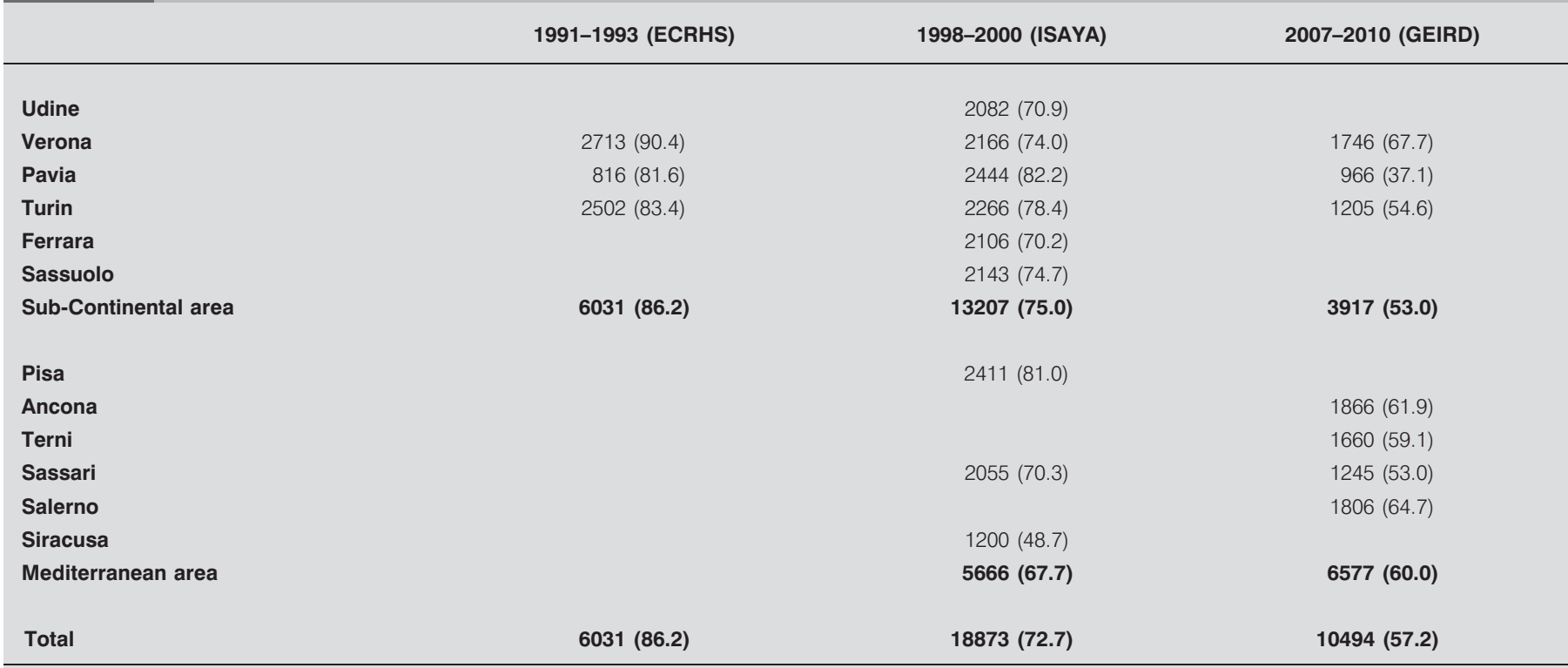

Data are presented as $n$ (response rate in \%). Figures in bold are summations of data from groups of centres. ECRHS: European Community Respiratory Health Survey; ISAYA: Italian Study on Asthma in Young Adults; GEIRD: Gene Environment Interactions in Respiratory Diseases.

The mean percentage of females was 54.1\% (95\% CI 51.8-56.4\%) in subjects with asthma and $48.9 \%$ (95\% CI $47.7-50.0 \%$ ) in subjects with allergic rhinitis. The sex composition did not show any statistical significant variation during the period of the study (data not shown) both in asthmatic and allergic subjects.

Crude and adjusted centre-specific prevalence rates of respiratory outcomes, by study and climatic area, are reported in online supplementary table S1a-e.

Temporal change in prevalence of current asthma, allergic rhinitis and asthma-like symptoms in Italy

In the four centres (Pavia, Sassari, Turin and Verona) that participated in at least two studies, the overall adjusted prevalence (fig. 2) of allergic rhinitis and wheezing increased continuously between 1991-1993 to 2007-2010, while the prevalence of current asthma, shortness of breath and tightness in the chest was almost stable during the 1990s, and then it steadily increased between 1998-2000 and 2007-2010.

The analysis of temporal trends (table 4), after adjusting for centre and other confounders, showed no significant variation in the risk of reporting asthma or asthma-like symptoms from 1991-1993 to 1998-2000, except for shortness of breath, whose prevalence decreased (RR $0.72,95 \%$ CI $0.62-0.84 ; \mathrm{p}<0.001$ ). However, in the subsequent decade, the prevalence of current asthma increased by $38 \%$ (RR 1.38, 95\% CI 1.19-1.59) and the prevalence of all asthma-like symptoms from $18 \%$ to $45 \%$. The

TABLE 2 Distribution of sex, age, smoking habits and type of contact for subjects who participated in the three respiratory surveys carried out in Italy between 1991 and 2010

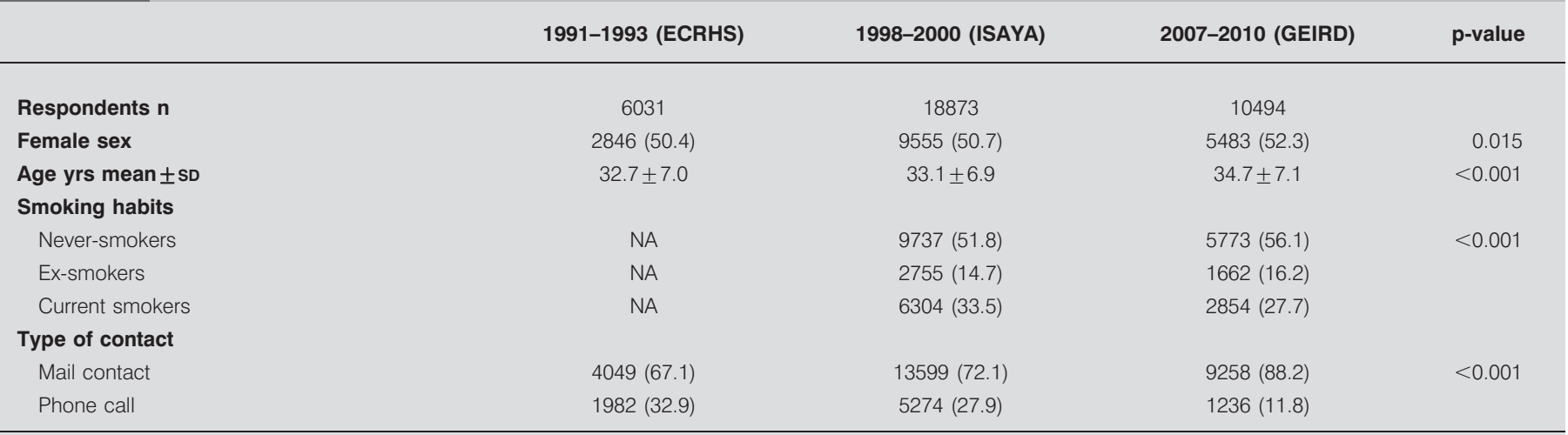

Data are presented as n (\%), unless otherwise stated. ECRHS: European Community Respiratory Health Survey; ISAYA: Italian Study on Asthma in Young Adults; GEIRD: Gene Environment Interactions in Respiratory Diseases. NA: information not available in the ECRHS survey. 


\begin{tabular}{|c|c|c|c|c|}
\hline TABLE 3 & \multicolumn{4}{|c|}{$\begin{array}{l}\text { Medians (ranges) of the adjusted }{ }^{\#} \text { prevalences of current asthma, allergic rhinitis, wheezing, shortness of breath and } \\
\text { tightness in the chest in the two main climatic regions (Sub-Continental and Mediterranean) and in the whole of Italy, } \\
\text { estimated in the centres that participated in the three respiratory surveys between } 1991 \text { and } 2010\end{array}$} \\
\hline \multicolumn{2}{|c|}{ Respondents n } & 6031 & 18873 & 10494 \\
\hline \multicolumn{5}{|c|}{ Current asthma } \\
\hline \multicolumn{2}{|c|}{ Mediterranean } & NA & $5.4(5.2-6.5)$ & $7.3(4.9-8.0)$ \\
\hline \multicolumn{2}{|c|}{ Overall } & $4.1(3.3-5.0)$ & $4.6(3.9-6.5)$ & $6.6(4.5-8.0)$ \\
\hline \multicolumn{5}{|c|}{ Allergic rhinitis } \\
\hline \multicolumn{2}{|c|}{ Sub-Continental } & $16.8(13.8-17.0)$ & $18.7(14.7-20.8)$ & $24.2(23.1-25.6)$ \\
\hline \multicolumn{2}{|c|}{ Mediterranean } & NA & $19.4(14.7-24.3)$ & $26.6(24.6-28.8)$ \\
\hline \multicolumn{2}{|l|}{ Overall } & $16.8(13.8-17.0)$ & $19.4(14.7-24.3)$ & $25.8(23.1-28.8)$ \\
\hline \multicolumn{5}{|c|}{ Shortness of breath } \\
\hline \multicolumn{2}{|c|}{ Sub-Continental } & $7.7(5.7-9.3)$ & $5.4(4.3-6.4)$ & $8.5(7.6-10.4)$ \\
\hline \multicolumn{2}{|c|}{ Mediterranean } & NA & $6.9(6.4-8.6)$ & $8.8(8.0-10.6)$ \\
\hline \multicolumn{2}{|l|}{ Overall } & $7.7(5.7-9.3)$ & $5.7(4.3-8.6)$ & $8.5(7.6-10.6)$ \\
\hline \multicolumn{5}{|c|}{ Tightness in the chest } \\
\hline \multicolumn{2}{|c|}{ Sub-Continental } & $7.6(6.4-10.1)$ & $7.6(5.9-8.0)$ & $10.2(7.7-10.9)$ \\
\hline \multicolumn{2}{|c|}{ Mediterranean } & NA & $8.8(7.9-11.1)$ & $10.3(8.3-13.2)$ \\
\hline \multicolumn{2}{|l|}{ Overall } & $7.6(6.4-10.1)$ & $7.8(5.9-11.1)$ & $10.2(7.7-13.2)$ \\
\hline
\end{tabular}

Data are presented as median (range) percentages, unless otherwise stated. ECRHS: European Community Respiratory Health Survey; ISAYA: Italian Study on Asthma in Young Adults; GEIRD: Gene Environment Interactions in Respiratory Diseases. NA: information not available because no centre in the Mediterranean area participated in the ECRHS survey. " : adjusted for sex, age, season of response, type of contact (mail/phone) and percentile rank of cumulative response.

prevalence of allergic rhinitis showed a statistically significant $16 \%$ increase in the first period and a further $27 \%$ increase in the last decade.

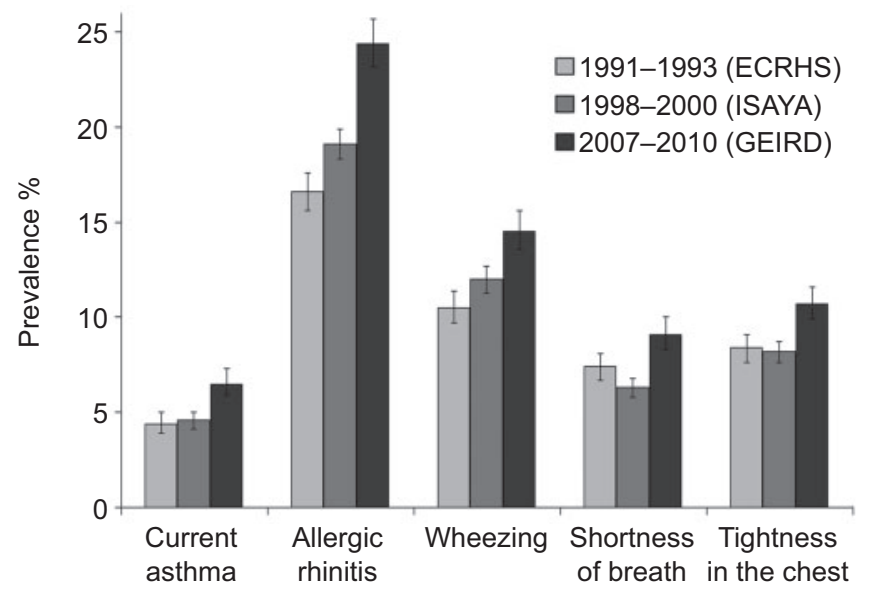

FIGURE 2. Overall mean prevalence, adjusted for sex, age, season of response, type of contact (mail/phone) and percentile rank of cumulative response, of current asthma, allergic rhinitis, wheezing, shortness of breath and tightness in the chest in the four centres (Pavia, Sassari, Turin and Verona) that participated in at least two surveys.
No interaction was found between calendar period, sex or age and current asthma prevalence. The temporal trends in prevalence of respiratory outcomes were similar in males and females and among different age groups (online supplementary tables S2 and S3).

\section{Temporal change in the prevalence of current asthma in subjects with and without allergic rhinitis}

In the four centres with at least two repeated surveys, 3,977 $(19.9 \%)$ subjects reported hay fever or allergic rhinitis on at least one occasion in either survey and 16,007 (80.1\%) did not. The prevalence of current asthma was $17.8 \%$ in the former and $2.0 \%$ in the latter group $(\mathrm{p}<0.001)$. Overall, $31.5 \%(95 \% \mathrm{CI}$ $28.6 \%-34.3 \%$ ) of all the current asthmatics did not report allergic rhinitis.

The trend in asthma prevalence was not influenced by the presence of allergic rhinitis in the period from 1991-2000; while in the last decade, the prevalence of current asthma was particularly high in subjects who did not have allergic rhinitis (fig. 3). In this group, there was a statistically significant increase of more than $36 \%$ (RR 1.36, 95\% CI 1.04-1.78) in the prevalence of asthma in 2007-2010 with respect to 1998-2000.

\section{Temporal change in the use of asthma medicine among asthmatics}

In the four centres with at least two repeated surveys, the percentages of subjects with current asthma who reported 
TABLE 4 Temporal changes in the prevalence of respiratory outcomes in the four centres (Pavia, Sassari, Turin, and Verona) that participated in at least two surveys

$\begin{array}{lc}\text { Current asthma } & 0.94(0.79-1.13) \\ \text { Allergic rhinitis } & 1.16(1.07-1.26)^{\star *} \\ \text { Wheezing } & 1.01(0.90-1.13) \\ \text { Shortness of breath } & 0.72(0.62-0.84)^{\star *} \\ \text { Tightness in the chest } & 0.91(0.79-1.04)\end{array}$

$1.38(1.19-1.59)^{* *}$

$1.27(1.19-1.36)^{\star \star}$

$1.18(1.07-1.28)^{* *}$

$1.45(1.28-1.63)^{\star *}$

$1.26(1.13-1.40)^{\star \star}$

Data are presented as relative risk $(95 \% \mathrm{Cl})$, obtained through Poisson regression models with robust estimates of the standard errors, adjusting for sex, age, season of response, type of contact (mail/phone) and percentile rank of cumulative response. ISAYA: Italian Study on Asthma in Young Adults; ECRHS: European Community Respiratory Health Survey; GEIRD: Gene Environment Interactions in Respiratory Diseases. **: $p \leqslant 0.001$

being on treatment were $48.0 \%$ (122 out of 254 ), $64.1 \%$ (266 out of 415 ) and $58.1 \%$ (211 out of 363) in 1991-1993, 1998-2000 and 2007-2010, respectively.

After adjusting for potential confounders, the maximum increase in asthma treatment among asthmatics was registered at the end of the 1990s (1998-2000 versus 1991-1993: RR 1.16, 95\% CI 0.97-1.38), while this proportion slightly decreased afterwards (table 5).

\section{Sensitivity analysis}

When the analyses of time trends were limited to same fixed cumulative response rates in each centre and in each study, the results were consistent with those previously presented (data not shown).

\section{DISCUSSION}

This analysis was aimed at assessing temporal changes in prevalence of current asthma, allergic rhinitis and asthma-like

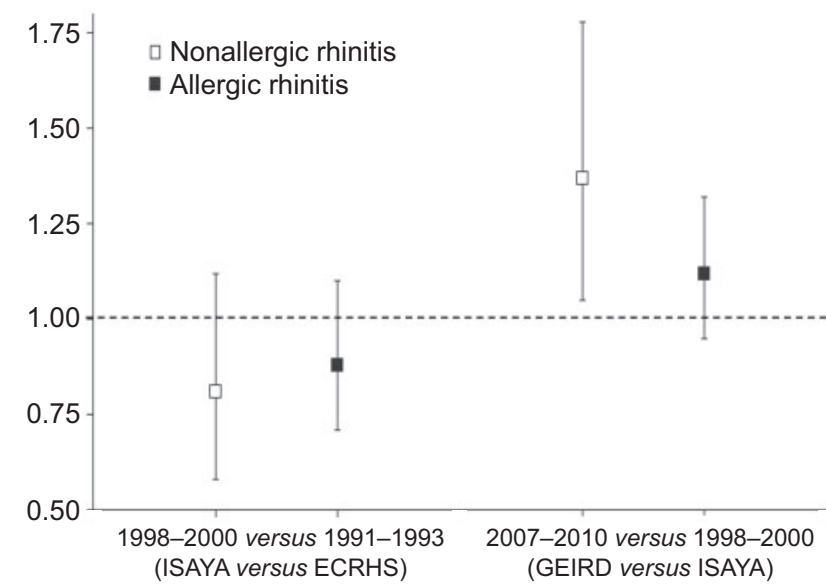

FIGURE 3. Temporal changes in the prevalence of current asthma among subjects with and without allergic rhinitis in the four centres (Pavia, Sassari, Turin and Verona) that participated in at least two surveys. Data are expressed as relative risk $(95 \% \mathrm{Cl})$, obtained through Poisson regression models, adjusting for sex, age, season of response, type of contact (mail/phone) and percentile rank of cumulative response. ISAYA: Italian Study on Asthma in Young Adults; ECRHS: European Community Respiratory Health Survey; GEIRD: Gene Environment Interactions in Respiratory Diseases. symptoms in Italy. Data from three multicentre studies, carried out between January 1991 and December 2010, that employed the same study design and the same methods, were used.

The main findings are as follows. 1) The asthma epidemic is not over in Italy. The prevalence of current asthma has increased by about $38 \%$ in the first decade of the third millennium, reaching an overall prevalence of $6.6 \%$. 2) The increase in asthma does not reflect only changes in diagnostic and treatment practices, as it is paralleled by a similar increase in asthma-like symptom prevalence. 3) The increase in asthma prevalence mainly concerns subjects who did not report allergic rhinitis. 4) The proportion of asthmatics undergoing treatment increased in the 1990s and then slightly decreased. 5) The prevalence of allergic rhinitis has increased continuously since 1991-1993, affecting more than one out of four young adults in Italy at the end of 2010.

\section{The asthma epidemic in Italy}

At the end of the 1990s, the few available data on the national [7] and international [8, 26] prevalence of asthma in adults seemed to suggest that the epidemic of asthma, which started in the 1950s, had reached a plateau and the trend in prevalence was fading away or even reversing.

Our analysis, which considers changes in prevalence from 1991 to 2010, documents that in Italy, the prevalence of asthma is on the increase again after an apparent period of stability at the end of the 1990s. The increase in the prevalence of reported asthma attacks and/or use of asthma medications is paralleled by a similar or stronger increase in the prevalence of asthmalike symptoms and allergic rhinitis. Hence, our findings strongly suggest that the asthma epidemic is not over. It is rather unlikely that this conclusion could be due to an artefact, because our prevalence estimates were adjusted for the difference between studies in potential confounding factors and in response rates. Furthermore, the sensitivity analyses confirmed the stability of our results under a wide spectrum of conditions.

The increase in asthma prevalence in the past 20 years was substantial. In 1991-1993, the median prevalence recorded in the three Italian centres that participated in the ECRHS survey was $4.1 \%$, ranging $3.3-5.0 \%$. According to this figure, Italy was classified as a country with a low-medium prevalence of asthma. 20 yrs later, when exactly the same questions were 


\begin{tabular}{|c|c|c|c|c|c|}
\hline \multirow[t]{3}{*}{ TABLE 5} & \multicolumn{5}{|c|}{$\begin{array}{l}\text { Temporal changes in the use of asthma medications among subjects with current asthma, in the centres (Pavia, } \\
\text { Sassari, Turin, and Verona) that participated in at least two surveys }\end{array}$} \\
\hline & & \multicolumn{2}{|c|}{$\begin{array}{l}\text { 1998-2000 versus 1991-1993 (ISAYA versus } \\
\text { ECRHS) }\end{array}$} & \multicolumn{2}{|c|}{$\begin{array}{l}\text { 2007-2010 versus 1998-2000 (GEIRD versus } \\
\text { ISAYA) }\end{array}$} \\
\hline & & RR $(95 \% \mathrm{Cl})$ & $p$-value & RR $(95 \% \mathrm{Cl})$ & $p$-value \\
\hline
\end{tabular}

administered in the frame of the GEIRD study, the median prevalence was $6.6 \%$, ranging $4.5-8.0 \%$. Interestingly, a crosssectional survey performed by the Italian College of General Practitioners in December 2009, found similar prevalence values [27].

Time trends in asthma prevalence were similar in males and females and in different age groups, as suggested by the absence of a statistically significant interaction between time, age and sex.

The greatest increase in asthma prevalence was observed after 1998, while the trend showed an apparent plateau before that year $[7,8]$. The upward trend in the prevalence of asthma, is consistent with other studies [3, 11, 12, 26, 27] and occurred while there was a concomitant substantial decrease in the percentage of current smokers. It may be the result of an increase in either the incidence and/or the persistence of the disease. The cross-sectional design of our study does not permit us to disentangle these two components of asthma. However, as asthma persistence is largely determined by the inability to achieve good control and/or to have a proper management of the disease, there is no reason to speculate that this component had a primary role in explaining the increase in prevalence (in general, new asthma medications and tools have improved the management of asthma in recent decades). Furthermore, the fact that in our study the prevalence of "lifelong asthma" had the same temporal increase as the prevalence of "current asthma" (data not shown) suggests that the observed trend in prevalence is likely to be due to a rise in asthma incidence over time. What the causes of the increase in asthma prevalence are is still an open question. Proposed contributing factors have included exposure to air pollution, infections and microbial substances in the environment [3]. Obesity may be another risk factor [30].

Our results confirm [24] that the prevalence of asthma and asthma-like symptoms in Italy is higher in the Mediterranean than in the Sub-Continental area. This difference could reflect the different exposures to outdoor and indoor allergens, or the difference in ozone concentration, which tends to reach higher levels at higher temperatures, or to the interaction between higher temperatures and pollution [20].

\section{The prevalence of asthma has significantly increased in non-allergic subjects}

Due to the strong association of asthma with atopic diseases reported in several studies [31] the role of non-atopic asthma has generally been underestimated [32], even if its prevalence in some countries is similar to [33], or even greater than, that of atopic asthma [34-36]. Only few studies have addressed the temporal variation of asthma prevalence in atopic and nonatopic subjects. In a study on Danish children [37], the prevalence of current asthma increased significantly from 1986 to 2001 and this was due primarily to non-atopic asthma. A Scottish study on adults reported that the prevalence of asthma increased from 1972-1976 to 1996 only in subjects without hay fever [38]. In a Swiss study, the decline in asthma consultations that occurred between 1999 and 2005 was attributed to the decline in prevalence of the allergic component of asthma [39].

Our results document that in Italy, too, non-atopic asthma affects about one out of three current asthmatics and that the greatest increase in asthma prevalence observed in the past $20 \mathrm{yrs}$ is due to nonallergic asthma.

As atopic and non-atopic asthma show distinct patterns of risk factors, the striking increase in the prevalence of non-atopic asthma in the past 20 yrs may reflect changes in population exposures, particularly in early-life exposures to viruses and/ or environmental pollutants, which are strong risk factors for non-atopic asthma [40].

Some caution is needed in interpreting this finding as objective indicators of atopy (such as skin-prick tests) are lacking. We considered an associated symptom (hay fever), which is often used when skin test data are not available [38] and has been shown to be a good marker of atopy [41-43].

\section{Allergic rhinitis has increased continuously}

The worldwide incidence and prevalence of allergic rhinitis has been on the rise since at least 1990 [4, 7, 13, 44] in almost all westernised countries. Whether the upward trend is due to increasing air pollution, indoor environmental factors, improved hygiene practices, geo-climatic factors or all of the above is open to the debate.

Our findings document that the prevalence of allergic rhinitis has also increased continuously in Italy in the past $20 \mathrm{yrs}$, affecting more than one out of four young adults at the end of 2010.

In 1991-1993, the percentage of sensitised subjects among those who reported allergic rhinitis in the frame of the Italian arm of ECRHS was 79\% [41]. Provided that this proportion has not 
changed in the past decade, it can be speculated that the increase in the prevalence of allergic rhinitis is the result of an increase in sensitisation among Italian adults.

\section{Temporal change in the use of asthma drugs}

Since 1990, national studies have shown that in Italy a considerable percentage of subjects with current asthma are not treated. Even when only patients with severe asthma were considered, the percentage of untreated patients was still high [45]. A recent analysis of the international ECRHS database documented that Italy is the country with the highest percentage of uncontrolled asthmatics [46], because of the high prevalence of untreated asthma. Our findings concerning temporal variations in the proportion of asthmatics in treatment, showed a slight increase at the end of the 1990s, when particular attention to the dissemination of the Global Initiative for Asthma (GINA) guidelines [47] probably reached a peak, followed by a minor decrease in more recent years. Even though these variations were not statistically significant, they seem to underline that 20 yrs after the publication of the GINA guidelines, the management of asthma still needs to be improved.

\section{Limits and merits of the study}

The present study benefited from applying the same study design, sampling frame, study protocol and administering the same questionnaire to three large, multicentre general population random samples of identical age, surveyed 10 yrs apart. Prevalence was computed according to self-reported symptoms and asthma attacks collected from validated international questionnaires [18]. Unlike diagnoses or medication use, selfreported symptoms are less influenced by diagnostic activity. Accordingly, it is extremely likely that temporal changes in symptoms reflect a trend in prevalence rather than changes in healthcare practice.

The Italian centres involved in the study were not chosen randomly but on the basis of the availability of research teams able to carry out the survey. However, the study areas are spread all over Italy and well represent the geographical (North versus South, Sub-Continental versus Mediterranean) features of the country. Furthermore, in order to avoid temporal changes in prevalence being biased by differences in geographical locations, the assessment of the time trend was limited only to those centres in which the survey was repeated at least twice.

In the past $30 \mathrm{yrs}$, participation rates in epidemiological studies have been declining throughout the industrialised world [48]. In Italy, as in other European countries, the average response rate decreased by about $15 \%$ every 10 yrs between 1991 and 2010. The decreased response rates over time may have biased our estimates. In fact, overestimation of symptom prevalence [19] in the latest surveys may have occurred, if the tendency to answer screening questionnaires is related to respiratory health. Fortunately, most empirical work in the last few years suggests that the decline in participation rates is not likely to have a substantial influence on exposure-disease associations or point estimates of the measure of interest [47]. Furthermore, our results were adjusted for an indicator of propensity to respond and the sensitivity analyses, which compared the data obtained with the same response rates in the three studies, confirmed the main results.

\section{Conclusions}

To sum up, we analysed the data of three large surveys carried out in Italy from 1990 to 2010, using the same protocol and methods, headed by the same research team. We found that asthma prevalence had increased by over 35\%, and this increase was mainly due to non-atopic asthma. A similar or even greater increase was documented for asthma-like symptoms and allergic rhinitis. These findings demonstrate the increasing social burden and impact of asthma and allergic rhinitis and enable healthcare providers and policy makers to plan effective clinical and public health strategies. Future research should focus on factors (allergen and pollutant outdoor concentrations, virus infections) that are potentially able to explain these temporal trends.

\section{SUPPORT STATEMENT}

The GEIRD project was funded by the Cariverona Foundation (Verona, Italy) and by the Italian Ministry of Education, Universities and Research (MIUR).

\section{STATEMENT OF INTEREST}

A statement of interest for I. Cerveri can be found at www.erj. ersjournals.com/site/misc/statements.xhtml

\section{ACKNOWLEDGEMENTS}

The members of the GEIRD study group are as follows.

R. de Marco, G. Verlato, M.E. Zanolin, S. Accordini, O. Bortolami, M. Braggion, V. Cappa, L. Cazzoletti, P. Girardi, F. Locatelli, A. Marcon, E. Montoli, M. Rava, R. Vesentini (Unit of Epidemiology and Medical Statistics, University of Verona, Verona, Italy); M. Ferrari, L. Donatelli, C. Posenato, V. Lo Cascio (Section of Internal Medicine, University of Verona); L. Perbellini, M. Olivieri, J. D'Amato, E. Donatini, M. Martinelli (Unit of Occcupational Medicine, Azienda Ospedaliera "Istituti Ospitalieri di Verona", Verona); P.F. Pignatti, C. Bombieri, M.D. Bettin, E. Trabetti (Unit of Biology and Genetics, University of Verona); A. Poli, M. Nicolis, S. Sembeni (Unit of Hygiene and Preventive, Environmental and Occupational Medicine, University of Verona); L. Antonicelli, F. Bonifazi (Dept of Internal Medicine, Immuno-Allergic and Respiratory Diseases, Ospedali Riuniti di Ancona, Ancona); F. Attena, V. Galdo (Dept of Public, Clinical and Preventive Medicine, II University of Naples, Naples); V. Bellia, S. Battaglia (Dept of Medicine, Pneumology, Physiology and Human Nutrition, University of Palermo, Palermo); I. Cerveri, A.G. Corsico, F. Albicini, A. Grosso (Division of Respiratory Diseases, IRCCS Policlinico "San Matteo", University of Pavia, Pavia), A. Marinoni, S. Villani, V. Ferretti (Dept of Health Sciences, University of Pavia); L. Casali, A. Miniucchi (Dept of Internal Medicine, Section of Respiratory Diseases, University of Perugia, Perugia); L. Briziarelli, M. Marcarelli (Dept of Medical-Surgical Specialties and Public Health, University of Perugia); M.G. Panico (National Health Service, Epidemiology Unit, ASL 2, Salerno); P. Pirina, A.G. Fois, F. Becciu, A. Deledda, V. Spada (Institute of Respiratory Diseases, University of Sassari, Sassari); M. Bugiani, A. Carosso, P. Piccioni, G. Castiglioni (National Health Service, CPA-ASL TO2, Unit of Respiratory Medicine and Allergology, Turin); R. Bono, R. Tassinari, V. Romanazzi (Dept of Public Health and Microbiology, University of Turin, Turin); G. Rolla, E. Heffler (Dept of Biomedical Sciences and Human Oncology, University of Turin); E. Migliore (Centre of Oncologic Prevention, Turin).

\section{REFERENCES}

1 World Health Organization. Global Surveillance, Prevention and Control of Chronic Respiratory Diseases: a Comprehensive Approach. Geneva, World Health Organization, 2007. 
2 Bahadori K, Doyle-Waters MM, Marra C, et al. Economic burden of asthma: a systematic review. BMC Pulm Med 2009; 19: 9-24.

3 Eder W, Ege MJ, von Mutius E. The asthma epidemic. N Engl J Med 2006; 355: 2226-2235.

4 Asher MI, Montefort S, Björkstén B, et al. Worldwide time trends in the prevalence of symptoms of asthma, allergic rhinoconjunctivitis, and eczema in childhood: ISAAC Phases One and Three repeat multicountry cross-sectional surveys. Lancet 2007; 368: 733-743.

5 Ng Man Kwong G, Proctor A, Billings C, et al. Increasing prevalence of asthma diagnosis and symptoms in children is confined to mild symptoms. Thorax 2001; 56: 312-314.

6 Shamssain M. Trends in the prevalence and severity of asthma, rhinitis and atopic eczema in 6- to 7- and 13- to 14-yr-old children from the north-east of England. Pediatr Allergy Immunol 2007; 18: 149-153.

7 Verlato G, Corsico A, Villani S, et al. Is the prevalence of adult asthma and allergic rhinitis still increasing? Results of an Italian study. J Allergy Clin Immunol 2003; 111: 1232-1238.

8 Chinn S, Jarvis D, Burney P, et al. Increase in diagnosed asthma but not in symptoms in the European Community Respiratory Health Survey. Thorax 2004; 59: 646-651.

9 Ekerljung L, Andersson A, Sundblad BM, et al. Has the increase in the prevalence of asthma and respiratory symptoms reached a plateau in Stockholm, Sweden? Int J Tuberc Lung Dis 2010; 14: 764-771.

10 Lötvall J, Ekerljung L, Rönmark EP, et al. West Sweden Asthma Study: prevalence trends over the last 18 years argues no recent increase in asthma. Respir Res 2009; 12: 10-94.

11 Anandan C, Nurmatov U, van Schayck OC, et al. Is the prevalence of asthma declining? Systematic review of epidemiological studies. Allergy 2010; 65: 152-167.

12 Gershon AS, Guan J, Wang C, et al. Trends in asthma prevalence and incidence in Ontario, Canada, 1996-2005: a population study. Am J Epidemiol 2010; 172: 728-736.

13 Braunstahl GJ. United airways concept: what does it teach us about systemic inflammation in airways disease? Proc Am Thorac Soc 2009; 6: 652-654.

14 Lee SL, Wong W, Lau YL. Increasing prevalence of allergic rhinitis but not asthma among children in Hong Kong from 1995 to 2001 (Phase 3 International Study of Asthma and Allergies in Childhood). Pediatr Allergy Immunol 2004; 15: 72-78.

15 Bråbäck L, Hjern A, Rasmussen F. Trends in asthma, allergic rhinitis and eczema among Swedish conscripts from farming and non-farming environments. A nationwide study over three decades. Clin Exp Allergy 2004; 34: 38-43.

16 Simpson CR, Newton J, Hippisley-Cox J, et al. Incidence and prevalence of multiple allergic disorders recorded in a national primary care database. J R Soc Med 2008; 101: 558-563.

17 Anderson HR. Prevalence of asthma. BMJ 2005; 330: 1037-1038.

18 European Community Respiratory Health Survey (ECRHS) Steering committee. Variations in the prevalence of respiratory symptoms, self-reported asthma attacks, and use of asthma medication in the European Community Respiratory Health Survey (ECRHS). Eur Respir J 1996; 9: 687-695.

19 de Marco R, Verlato G, Zanolin ME, et al. Nonresponse bias in EC Respiratory Health Survey in Italy. Eur Respir J 1994; 7: 2139-2145.

20 de Marco R, Poli A, Ferrari M, et al. The impact of climate and traffic-related $\mathrm{NO}_{2}$ on the prevalence of asthma and allergic rhinitis in Italy. Clin Exp Allergy 2002; 32: 1405-1412.

21 de Marco R, Accordini S, Antonicelli L, et al. The Gene Environmental Interactions in Respiratory Diseases (GEIRD) Project. Int Arch Allergy Immunol 2010; 152: 255-263.

22 de Marco R, Zanolin ME, Accordini S, et al. A new questionnaire for the repeat of the first stage of the European Community Respiratory Health Survey: a pilot study. Eur Respir J 1999; 14: 1044-1048.
23 Ronmark E, Lundqvist A, Lundback B, et al. Non-responders to a postal questionnaire on respiratory symptoms and diseases. Eur $J$ Epidemiol 1999; 15: 293-299.

24 Zanolin ME, Pattaro C, Corsico A, et al. The role of climate on the geographic variability of asthma, allergic rhinitis and respiratory symptoms: results from the Italian study of asthma in young adults. Allergy 2004; 59: 306-314.

25 Zou G. A modified Poisson regression approach to prospective studies with binary data. Am J Epidemiol 2004; 159: 702-706.

26 Woods RK, Walters EH, Wharton C, et al. The rising prevalence of asthma in young Melbourne adults is associated with improvement in treatment. Ann Allergy Asthma Immunol 2001; 87: 117-123.

27 Cazzola M, Puxeddu E, Bettoncelli G, et al. The prevalence of asthma and COPD in Italy: a practice-based study. Respir Med 2011; 105: 386-391.

28 Garner R, Kohen D. Changes in the prevalence of asthma among Canadian children. Health Rep 2008; 19: 45-50.

29 Bjerg A, Sandström T, Lundbäck B, et al. Time trends in asthma and wheeze in Swedish children 1996-2006: prevalence and risk factors by sex. Allergy 2010; 65: 48-55.

30 Ford ES. The epidemiology of obesity and asthma. J Allergy Clin Immunol 2005; 115: 897-909.

31 Antó JM, Sunyer J, Basagaña X, et al. Risk factors of new-onset asthma in adults: a population-based international cohort study. Allergy 2010; 65: 1021-1030.

32 Pearce N, Pekkanen J, Beasley R. How much asthma is really attributable to atopy? Thorax 1999; 54: 268-272.

33 Kurukulaaratchy RJ, Fenn M, Mathews S, et al. Characterisation of atopic and non-atopic wheeze in 10 year old children. Thorax 2004; 59: 563-568.

34 Pereira MU, Sly PD, Pitrez PM, et al. Nonatopic asthma is associated with helminth infections and bronchiolitis in poor children. Eur Respir J 2007; 29: 1154-1160.

35 Moncayo AL, Vaca M, Oviedo G, et al. Risk factors for atopic and non atopic asthma in a rural area of Ecuador. Thorax 2010; 65: 409-416.

36 Ronchetti R, Jesenak M, Ronchetti F, et al. Is asthma caused by atopy (positive skin prick tests)? Epidemiologic evidence suggests a negative answer. Inflamm Allergy Drug Targets 2010; 9: 91-96.

37 Thomsen SF, Ulrik CS, Larsen K, et al. Change in prevalence of asthma in Danish children and adolescents. Ann Allergy Asthma Immunol 2004; 92: 506-511.

38 Upton MN, McConnachie A, McSharry C, et al. Intergenerational 20 year trends in the prevalence of asthma and hay fever in adults: the Midspan family study surveys of parents and offspring. $B M J$ 2000; 321: 88-92.

39 Bollag U, Grize L, Braun-Fahrländer C. Is the ebb of asthma due to the decline of allergic asthma? A prospective consultation-based study by the Swiss Sentinel Surveillance Network, 1999-2005. Fam Pract 2009; 26: 96-101.

40 Janson C, Kalm-Stephens P, Foucard T, et al. Risk factors associated with allergic and non-allergic asthma in adolescents. Clin Respir J 2007; 1: 16-22.

41 Olivieri M, Verlato G, Corsico A, et al. Prevalence and features of allergic rhinitis in Italy. Allergy 2002; 57: 600-606.

42 Bugiani M, Carosso A, Migliore E, et al. Allergic rhinitis and asthma comorbidity in a survey of young adults in Italy. Allergy 2005; 60: 165-70.

43 Braun-Fahrländer C, Wüthrich B, Gassner M, et al. Validation of a rhinitis symptom questionnaire (ISAAC core questions) in a population of Swiss school children visiting the school health services. SCARPOL-team. Swiss Study on Childhood Allergy and Respiratory Symptom with respect to Air Pollution and Climate. International Study of Asthma and Allergies in Childhood. Pediatr Allergy Immunol 1997; 8: 75-82.

44 Bjerg A, Ekerljung L, Middelveld R, et al. Increased prevalence of symptoms of rhinitis but not of asthma between 1990 and 2008 in Swedish adults: comparisons of the ECRHS and GA ${ }^{2}$ LEN Surveys. PLOS ONE 2011; 6: e16082. 
45 Cerveri I, Zoia MC, Bugiani M, et al. Inadequate antiasthma drug use in the north of Italy. Eur Respir J 1997; 10: 2761-2765.

46 Cazzoletti L, Marcon A, Janson C, et al. Asthma control in Europe: a real-world evaluation based on an international populationbased study. J Allergy Clin Immunol 2007; 120: 1360-1367.
47 NHLBI/WHO Workshop Report: Global Strategy for Asthma Management and Prevention Issued January, 1995. NIH Publication No 02-3659. Bethesda, National Institutes for Health, 1995.

48 Galea S, Tracy M. Participation rates in epidemiological studies. Ann Epidemiol 2007; 17: 643-653. 